\title{
SARC-F Is Inaccurate to Identify Geriatric Rehabilitation Inpatients at Risk for Sarcopenia: RESORT
}

\author{
Lenore Dedeyne $^{a, b} \quad$ Esmee M. Reijnierse $^{b} \quad$ Jacob Pacifico $^{b} \quad$ Jacqueline E. Kay $^{c}$ \\ Patricia Maggs ${ }^{c}$ Sabine Verschueren ${ }^{d}$ Jos Tournoy ${ }^{a, e}$ Evelien Gielen ${ }^{\text {a, e }}$ \\ Wen Kwang Limb Andrea B. Maier ${ }^{b}$, \\ aDepartment of Public Health and Primary Care, Gerontology \& Geriatrics, KU Leuven, Leuven, Belgium; \\ ${ }^{b}$ Department of Medicine and Aged Care, @AgeMelbourne, The Royal Melbourne Hospital, The University of \\ Melbourne, Parkville, VIC, Australia; 'Department of Allied Health, Physiotherapy, The Royal Melbourne Hospital, \\ Parkville, VIC, Australia; ${ }^{d}$ Department of Rehabilitation Sciences, Musculoskeletal revalidation, KU Leuven, Leuven, \\ Belgium; 'Department of Geriatric Medicine, UZ Leuven, Leuven, Belgium; fDepartment of Human Movement \\ Sciences, @AgeAmsterdam, Vrije Universiteit Amsterdam, Amsterdam Movement Sciences, Amsterdam, The \\ Netherlands
}

\section{Keywords}

Sarcopenia · Geriatric assessment · Inpatients · Sensitivity and specificity

\begin{abstract}
Introduction: Sarcopenia is highly prevalent in geriatric rehabilitation inpatients; screening using the Strength, Assistance in walking, Rise from a chair, Climb stairs, Falls history questionnaire (SARC-F) has been recommended. This study assessed the diagnostic accuracy of the SARC-F in identifying sarcopenia according to the European Working Group on Sarcopenia in Older People (EWGSOP), EWGSOP2, and Asian Working Group for Sarcopenia (AWGS) definitions in geriatric rehabilitation inpatients. Methods: REStOring health of acutely unwell adulTs (RESORT) is an observational, longitudinal cohort of geriatric rehabilitation inpatients. The SARCF was completed for 2 time-points, status at preadmission (1 month before admission) and at admission; a score $\geq 4$ was considered at risk for sarcopenia. Muscle mass (bioelectrical impedance analysis), handgrip strength (handheld dyna-
\end{abstract}

mometry), and gait speed (4-m walk test) were measured at admission. Diagnostic accuracy was determined by sensitivity, specificity, and area under the curve (AUC). Results: The sarcopenia prevalence $(n=290$, median age 84.0 years [IQR $79.0-89.0$ ], $56.9 \%$ female) was $40.3 \%$ (EWGSOP1), $25.4 \%$ (EWGSOP2), and 38.8\% (AWGS). For preadmission and admission status, respectively, the SARC-F identified 67.9 and 82.1\% (EWGSOP), 66.0 and 81.0\% (EWGSOP2), and 67.5 and $81.6 \%$ (AWGS) inpatients at risk for sarcopenia. The SARC-F showed fair sensitivity (67-74\%), poor specificity (32-37\%), and poor AUC (0.411-0.474) to identify inpatients at risk for sarcopenia at preadmission status, and fair-good sensitivity (79-84\%), poor specificity (17-20\%), and poor AUC (0.4010.432 ) to identify inpatients at risk for sarcopenia at admission, according to EWGSOP, EWGSOP2, and AWGS definitions. Conclusion: The SARC-F showed poor diagnostic accuracy in identifying sarcopenia in geriatric rehabilitation inpatients. Assessment of sarcopenia is recommended without screening.

(C) 2021 The Author(s)

Published by S. Karger AG, Basel karger@karger.com

www.karger.com/ger

Karger $\stackrel{\text { ' }}{=}$

BOPEN ACCESS
(C) 2021 The Author(s)

Published by S. Karger AG, Basel

This is an Open Access article licensed under the Creative Commons Attribution-NonCommercial-4.0 International License (CC BY-NC) (http://www.karger.com/Services/OpenAccessLicense), applicable to the online version of the article only. Usage and distribution for commercial purposes requires written permission.
Correspondence to:

Andrea B. Maier, a.b.maier@vu.nl 


\section{Introduction}

The prevalence of sarcopenia, age-related low muscle mass and function, has been reported to be as high as $51 \%$ in geriatric rehabilitation patients [1]. Sarcopenia predisposes older adults to disability, functional decline, falls, fractures, and death [2-4]. In 2013, the Strength, Assistance in walking, Rise from a chair, Climb stairs, Falls history questionnaire (SARC-F) for rapid screening for sarcopenia was proposed [5] and supported by expert groups such as the European Working Group on Sarcopenia in Older People (EWGSOP) [6], the Society of Sarcopenia, Cachexia and Wasting Disorders (SCWD) [7], and the task force of the International Conference on Frailty and Sarcopenia Research (ICFSR) [8].

The SARC-F is a simple and inexpensive tool encompassing 5 self-reported questions related to muscle function. The SARC-F has been validated in various ethnic populations in the community [6,9-14], acute geriatric care [15], hospitalized older adults with hip fracture [16], geriatric outpatients [17], and in nursing homes [18, 19]. Independent of the setting, low-to-moderate sensitivity and high specificity were reported [6, 9-15, 17-19], except for hospitalized older adults with hip fracture (good sensitivity and fair specificity) [16]. To the best of our knowledge, the diagnostic accuracy of SARC-F, the ability of a test to discriminate between the risk of sarcopenia and no risk of sarcopenia, has not yet been assessed in geriatric rehabilitation inpatients. Geriatric rehabilitation inpatients are older adults with various primary diseases admitted to geriatric wards with the aim to restore functional capability.

This study aimed to assess the diagnostic accuracy [sensitivity, specificity, and area under receiver operator curve (AUC)] of the SARC-F in identifying inpatients at risk for sarcopenia according to the EWGSOP [20], which is the operational definition used in Australia and New Zealand [21], EWGSOP2 [6], and Asian Working Group for Sarcopenia (AWGS) [22] definitions in geriatric rehabilitation inpatients.

\section{Materials and Methods}

\section{Study Design}

The REStORing health of acutely unwell adulTs (RESORT) is an observational, longitudinal cohort of geriatric rehabilitation inpatients at a teaching hospital (Royal Melbourne Hospital, Melbourne, VIC, Australia) initiated in October 2017. All patients were assessed using a Comprehensive Geriatric Assessment (CGA) within $48 \mathrm{~h}$ of admission to geriatric rehabilitation by a multidisciplinary team of physicians, nurses, physiotherapists, occupation- al therapists, and dietitians. Written informed consent was obtained by the patient or a nominated proxy. Patients were excluded if they were palliative at admission or if the patient had no capacity to consent and there was no nominated proxy to consent. In the current analysis, patients were included based on data availability of the SARC-F screening tool and sarcopenia assessment. The SARC-F was introduced in July 2018. The study was approved by the Melbourne Health Human Research Ethics Committee (HREC/17/MH/103) and performed in accordance to the Declaration of Helsinki.

\section{Patient Characteristics}

Age, sex, and length of stay were obtained from medical records. Current living situation (living alone) and current smoking status were obtained from a survey completed by the patient and/ or carer. Standing height, up to the nearest $0.1 \mathrm{~cm}$, was measured without footwear if the patient was able to stand. If the patient was unable to stand, knee height was measured by a sliding caliper between knee and ankle joints positioned at $90^{\circ}$; the estimated height was then calculated using the Chumlea equation for Caucasian individuals [23]. Weight, up to the nearest $0.1 \mathrm{~kg}$, was measured on a calibrated weighing scale, weighing chair, or hoist. The body mass index was calculated by body weight divided by height squared $\left(\mathrm{kg} / \mathrm{m}^{2}\right)$. The Clinical Frailty Scale was measured on a scale from 1 (very fit) to 9 (terminally ill) [24]. Comorbidity was assessed with the Charlson Comorbidity Index (CCI), by quantifying the 19 co-morbid conditions on a 0-37 scale [25]. Cognitive impairment was evaluated by the presence of dementia or by a cognitive score below cut-off values of one of following tests: Mini-Mental State Examination (MMSE) $<24$ points, Montreal Cognitive Assessment (MOCA) $<26$ points, or Rowland Universal Dementia Assessment Scale (RUDAS) <23 points. Risk of malnutrition was assessed by the Malnutrition Screening Tool (MST) on a scale from 0 to 5 [26]. Functional independence status was examined by the Katz Activities of Daily Living (ADL) [27] and Lawton and Brody Instrumental Activities of Daily Living (IADL) [28]. Scores of ADLs and IADL ranged between $0-6$ and $0-8$ points, respectively, higher scores indicating higher level of independence.

\section{Risk of Sarcopenia}

The SARC-F questionnaire [5] was completed by a survey completed by the patient and/or carer. The questionnaire assesses 5 self-reported components: muscle strength, assistance with walking, rise from a chair, climb stairs, and falls history. The total score ranges from 0 to 10 points, with $0-2$ points for each component; higher scores indicating a higher risk of sarcopenia. A cut-off score of 4 points or higher defines risk of sarcopenia. Patients and/or their carer completed the SARC-F at admission to geriatric rehabilitation for both their preadmission status, describing the situation a month before hospital admission, and for their status at admission (at that moment) to geriatric rehabilitation. The risk for sarcopenia at preadmission was assessed to identify the predictive capacity of the SARC-F 1 month before hospitalization. The study sample for the present analyses included 318 patients for whom the SARC-F was completed. The SARC-F was performed independently of the sarcopenia assessment.

\section{Sarcopenia}

Sarcopenia was defined based on muscle mass and muscle function (strength and/or physical performance) in accordance to 
the EWGSOP definition [20], the revised EWGSOP2 definition [6], and the AWGS definition [22].

Direct-segmental multifrequency bioelectrical impedance analysis (DSM-BIA, InBody S10, Biospace Co., Ltd, Seoul, South Korea) was used to measure skeletal muscle mass and appendicular lean mass (ALM) in kilograms. BIA has been validated for assessing segmental and whole-body composition against dual-energy X-ray absorptiometry [29] and is more practical on hospital wards. BIA was not performed when the patient (1) had an electronic internal medical device or implant; or (2) plasters or bandages hindering the placing of the electrodes; or (3) amputation; or (4) admitted under contact isolation. Skeletal muscle mass index [30] was calculated by dividing skeletal muscle mass (kg) by height squared $\left(\mathrm{m}^{2}\right)$. ALM $(\mathrm{kg})$ was divided by height squared $\left(\mathrm{m}^{2}\right)$. Low muscle mass was defined as skeletal muscle mass index $\leq 10.75 \mathrm{~kg} / \mathrm{m}^{2}$ for males or $\leq 6.75 \mathrm{~kg} / \mathrm{m}^{2}$ for females for EWGSOP [20], ALM/height ${ }^{2}<7.0 \mathrm{~kg} / \mathrm{m}^{2}$ for males or $<5.5 \mathrm{~kg} / \mathrm{m}^{2}$ for females for EWGSOP2 [6], and ALM/height ${ }^{2} \leq 7.0 \mathrm{~kg} / \mathrm{m}^{2}$ for males or $<5.7$ $\mathrm{kg} / \mathrm{m}^{2}$ for females for AWGS [22].

Handgrip strength was measured with a handheld dynamometer (JAMAR, Sammons Preston, Inc., 119 Bolingbrook, IL, USA) in a seated position with elbows flexed at $90^{\circ}$, shoulders adducted, and forearms in a neutral position without support. The test was repeated 3 times on each side alternating and the maximal value was reported in kilograms [31]. The Short Physical Performance Battery was used to assess physical function on a scale of $0-12$ points [32]. Gait speed was measured over a 4-m course at a usual pace according to the Short Physical Performance Battery protocol. The test was repeated 2 times and the fastest time in seconds was converted to gait speed per second $(\mathrm{m} / \mathrm{s})$.

\section{Statistical Analyses}

Normally distributed continuous variables were presented as the mean and standard deviation and skewed distributed continuous variables were presented as the median with interquartile range (IQR). Normality of variables was checked with the ShapiroWilk test. Categorical variables were presented as a numerical value $(n)$ with percentages (\%).

The correlation between both SARC-F measures for preadmission status and at admission was assessed by the Spearman rank correlation. The correlation coefficient was interpreted as poor if $\leq 0.299$, fair if $\geq 0.3$ and $\leq 0.599$, moderate if $\geq 0.6$ and $\leq 0.799$, very strong if $\geq 0.8$. Using sarcopenia according to the EWGSOP, EWGSOP2, or AWGS criteria as the reference, the diagnostic accuracy of the SARC-F questionnaire was assessed through the sensitivity, specificity, and AUC. Sensitivity and specificity were classified as poor if $<50 \%$, fair if $>50 \%$ and $<80 \%$, good if $\geq 80 \%$. AUC was classified as poor if $<0.60$, fair $0.60-0.80$, and good $>0.80$. The SARC-F was considered valid when the diagnostic accuracy defined by AUC was $>0.80$. SARC-F was also analyzed as a continuous variable (range $0-10$ points) to examine the diagnostic accuracy of the tool and determine sensitivity/specificity of previously found suggested cut-off points 3 and $6[15,16,33]$.

Subgroup analyses were performed to assess the differences in diagnostic accuracy between the SARC-F completion in the presence of the patient or by the patient himself compared to the SARC-F completion by the carer without the patient.

Data analyses were performed using IBM SPSS Statistical package (IBM Corp. Released 2017 IBM SPSS Statistics for Windows, Version 25.0, Armonk, NY).
Table 1. Characteristics of geriatric rehabilitation inpatients at admission $(n=290)$

\begin{tabular}{|c|c|c|}
\hline Characteristic & $N$ & Total \\
\hline Age, years & 290 & $84.0(79.0-89.0)$ \\
\hline Female, $n(\%)$ & 290 & $165(56.9)$ \\
\hline LOS, days & 290 & $19.0(13-31)$ \\
\hline Living alone, $n(\%)$ & 283 & $118(41.7)$ \\
\hline Born in Australia, $n(\%)$ & 290 & $115(39.7)$ \\
\hline Current smoking, $n(\%)$ & 284 & $14(4.9)$ \\
\hline $\mathrm{BMI}, \mathrm{kg} / \mathrm{m}^{2}$ & 273 & $25.6(22.5-30.1)$ \\
\hline Frailty, score $0-9$ & 284 & $6(6-7)$ \\
\hline CCI, score $0-37$ & 290 & $2(1-4)$ \\
\hline Cognitive impairment, $n(\%)$ & 290 & $153(52.8)$ \\
\hline MST, score $0-5$ & 288 & $1(0-2)$ \\
\hline ADL, score $0-6$ & 284 & $1(1-2)$ \\
\hline IADL, score $0-8$ & 283 & $1(0-2)$ \\
\hline \multicolumn{3}{|l|}{ Sarcopenia measures } \\
\hline SMI, mean (SD), $\mathrm{kg} / \mathrm{m}^{2}$ & 263 & \\
\hline Females & 129 & $8.31(1.42)$ \\
\hline Males & 94 & $9.14(1.30)$ \\
\hline Unable, $n(\%)$ & & $40(15.2)$ \\
\hline $\mathrm{ALM} / \mathrm{h}^{2}, \mathrm{~kg} / \mathrm{m}^{2}$ & 263 & \\
\hline Females & 129 & $6.59[5.46-7.32]$ \\
\hline Males & 94 & $7.69[6.79-8.29]$ \\
\hline Unable, $n(\%)$ & & $40(15.2)$ \\
\hline $\mathrm{HGS}, \mathrm{kg}$ & 275 & \\
\hline Females & 139 & $13.0[10.0-18.0]$ \\
\hline Males & 111 & $21.0[16.0-26.0]$ \\
\hline Unable, $n(\%)$ & & $25(9.09)$ \\
\hline SPPB, score $0-12$ & 278 & $1[0-4]$ \\
\hline Four-meter walk test & 278 & \\
\hline Gait speed, m/s & 170 & $0.43[0.29-0.60]$ \\
\hline Unable, $n(\%)$ & & $108(38.8)$ \\
\hline
\end{tabular}

Data are presented as median [IQR] unless indicated otherwise. IQR, Interquartile range; SD, Standard deviation; LOS, Length of stay; BMI, Body mass index; CCI, Charlson Comorbidity index; MST, Malnutrition Screening Tool; ADL, Activities of Daily Living; IADL, Instrumental Activities of Daily Living; SMI, Skeletal Muscle mass Index; ALM, Appendicular lean mass; h, height; HGS, Handgrip strength; SPPB, Short Physical Performance Battery.

\section{Results}

Table 1 shows the characteristics of geriatric rehabilitation inpatients at admission. The SARC-F was completed for preadmission and admission status by 290 patients. The median age of this study sample was 84.0 years (IQR $79.0-89.0$ ), $56.9 \%$ of patients were female, $41.7 \%$ lived alone, $39.7 \%$ was born in Australia, and 52.8\% were cognitively impaired. Figure 1 shows the flow diagram of included patients. 


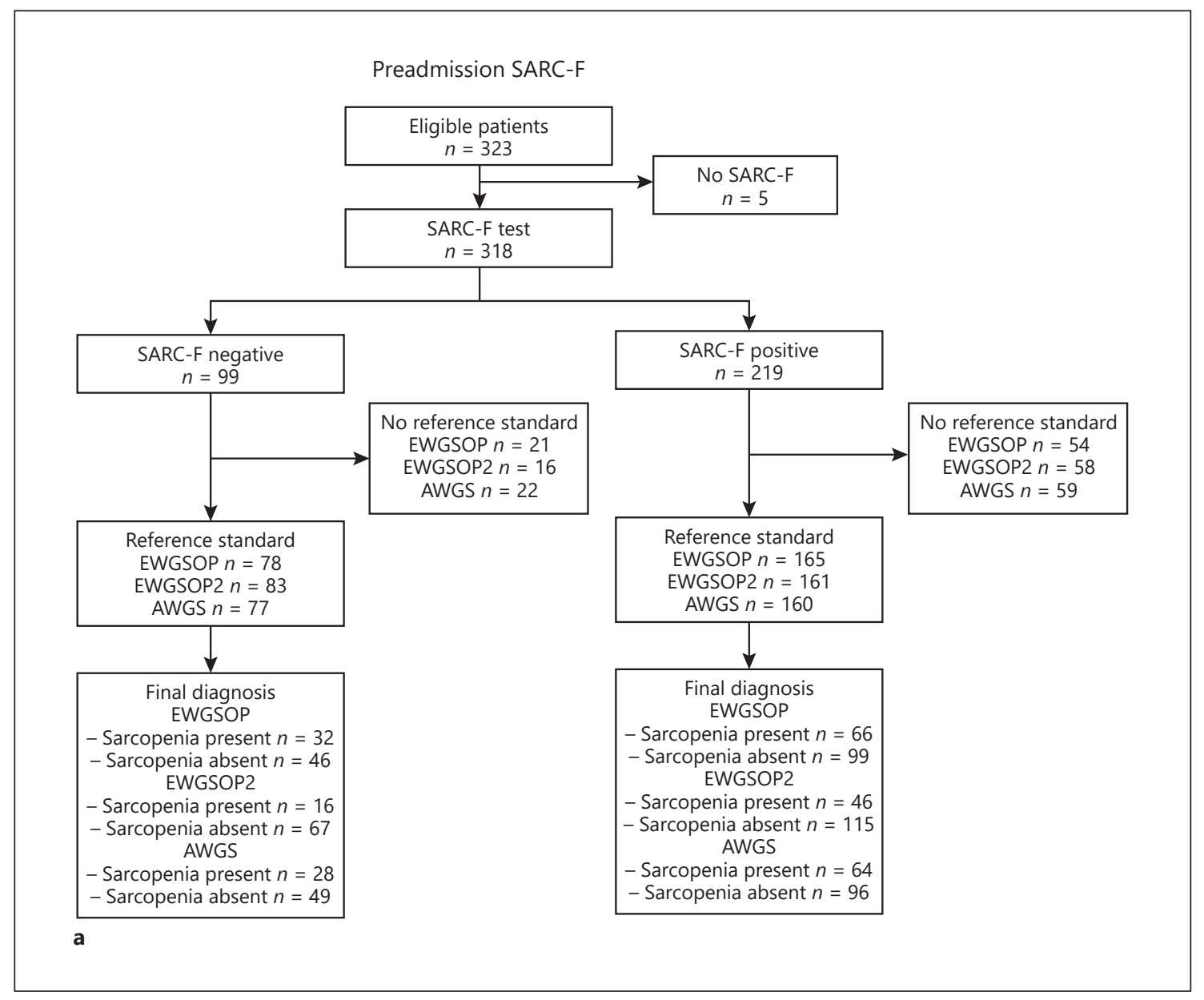

Fig. 1. Flow diagram of included patients according to STARD, stratified for SARC-F at preadmission status (before hospital admission) (a) and at admission status (at admission to geriatric rehabilitation) (b). SARC-F, Strength, Assistance in walking, Rise from a chair, Climb stairs, Falls history questionnaire. EWGSOP, European Working Group on Sarcopenia in Older People; AWGS, Asian Working Group for Sarcopenia; STARD, Standards for Reporting Diagnostic accuracy studies.

(Figure continued on next page.)

Table 2 shows that 71.0 and $83.8 \%$ of patients were screened at risk for sarcopenia for preadmission and admission status, respectively, including an overlap of $67.2 \%$. A Spearman's rank-order correlation showed a significant fair, positive correlation between SARC-F for preadmission and admission status $(r=0.480, p<0.001)$.

Table 3 shows that the prevalence of sarcopenia of geriatric rehabilitation inpatients was 40.3\% (EWGSOP), $25.4 \%$ (EWGSOP2), and $38.8 \%$ (AWGS). For preadmission status, the SARC-F identified $27.2 \%$ of patients at risk for sarcopenia with a false-positive rate of $40.7 \%$ and a false-negative rate of $13.2 \%$ (EWGSOP). For admission status, the SARC-F identified 33.2, 20.8 or $31.3 \%$ of patients at risk for sarcopenia with false-positive rates of $48.9,60.2$, or $50.2 \%$ and false-negative rates of $8.5,4.1$, or $6.5 \%$ (EWGSOP,
EWGSOP2, and AWGS, respectively). Figure 2 shows the distribution of SARC-F scores at preadmission and admission.

The diagnostic accuracy of the SARC-F (cut-off score 24) compared to EWGSOP, EWGSOP2, and AWGS is shown in Table 4. The SARC-F had fair sensitivity (67$74 \%$ ), poor specificity (32-37\%), and poor accuracy (0.4110.474 ) to identify inpatients at risk for sarcopenia for preadmission status compared to all 3 applied sarcopenia definitions. The SARC-F had fair to good sensitivity (79-84\%), poor specificity (17-20\%), and poor AUC (0.401-0.432) to identify inpatients at risk for sarcopenia for admission status compared to all 3 applied sarcopenia definitions.

The diagnostic accuracy of the SARC-F on a continuous scale was poor compared to EWGSOP, EWGSOP2 and 


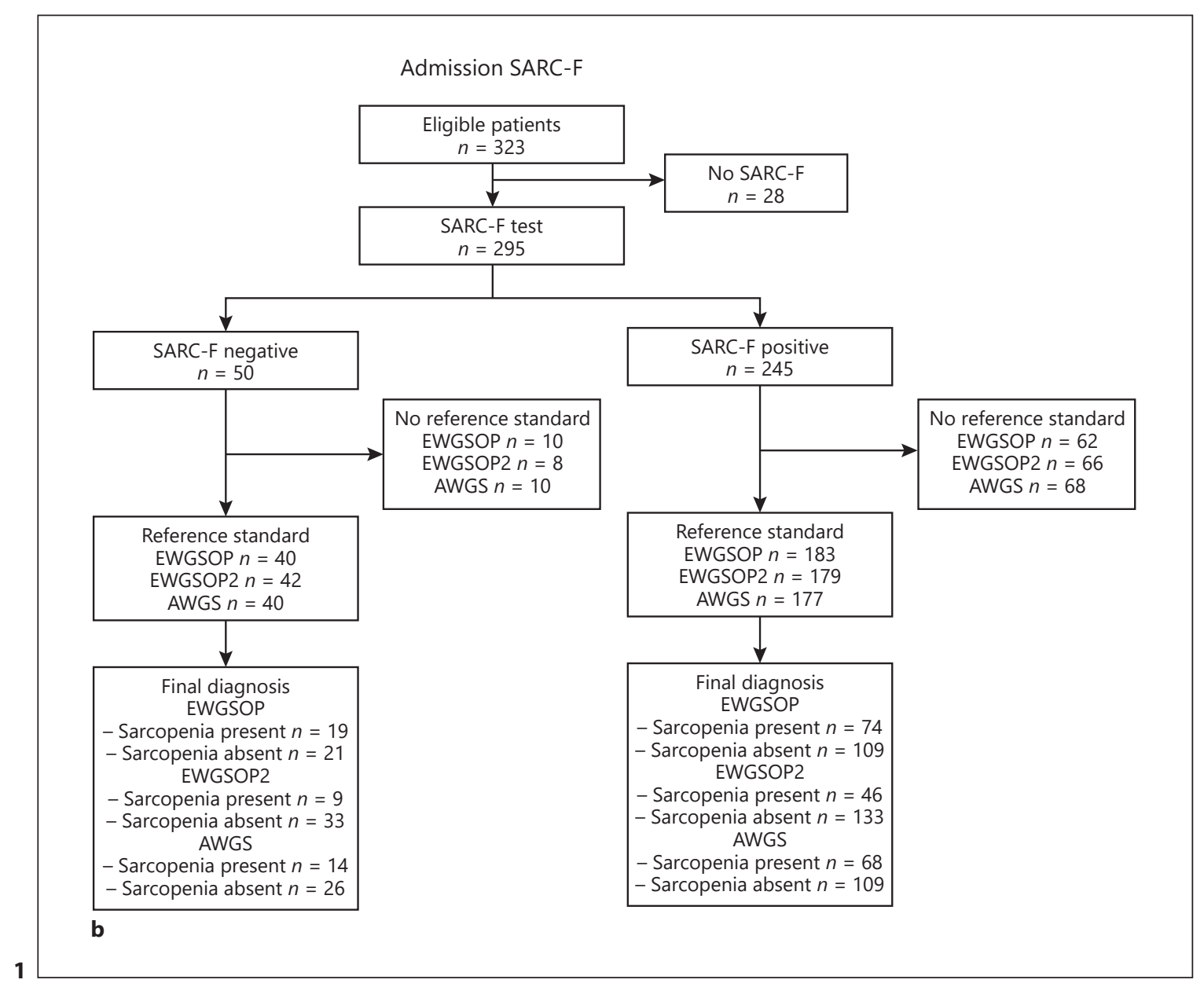

Table 2. SARC-F (cut-off $\geq 4$ ) screening for preadmission and admission status in geriatric rehabilitation inpatients

\begin{tabular}{lccr}
\hline SARC-F & \multicolumn{4}{l}{ SARC-F at admission, $n(\%)$} \\
\cline { 2 - 4 } & $\geq 4$ & $<$ & \multicolumn{1}{l}{ total } \\
\hline SARC-F & at & preadmission, $n(\%)$ & \\
$\geq 4$ & $195(67.2)$ & $11(3.8)$ & $206(71.0)$ \\
$<4$ & $48(16.6)$ & $36(12.4)$ & $84(29.0)$ \\
\hline Total & $243(83.8)$ & $47(16.2)$ & $290(100)$
\end{tabular}

SARC-F, Strength, Assistance in walking, Rise from a chair, Climb stairs, Falls history questionnaire.

AWGS (0.427-0.466). A cut-off point of 3 or 6 resulted in fair to good sensitivity and poor specificity for preadmission and admission status, except that the cut-off of 6 resulted in poor sensitivity and fair specificity for preadmission status (see online suppl. Table 1; for all online suppl. material, see www.karger.com/doi/10.1159/000516117).
The difference between the SARC-F completion in the presence of the patient or by the patient himself compared to the SARC-F completion by the carer without the patient is presented in online suppl. Tables 2 and 3. When completed in the presence of/by the patient, the SARC-F had fair sensitivity (59-75\%) and poor specificity $(21-41 \%)$ at both time points to identify inpatients at risk for sarcopenia compared to all 3 applied sarcopenia definitions. The survey completed without the patient resulted in fair to good sensitivity at preadmission (75-83\%) and good sensitivity at admission (91-100\%). The specificity was poor at preadmission and admission (8-32\%). The AUC was poor (0.443-0.573) at both time points, irrespective of who completed the SARC-F.

Online suppl. Tables 4 and 5 show the diagnostic accuracy in patients without cognitive impairment and online suppl. Tables 6 and 7 in patients with cognitive impairment. When completed in the presence of/by the patient, the SARC-F had fair sensitivity (51-74\% without 


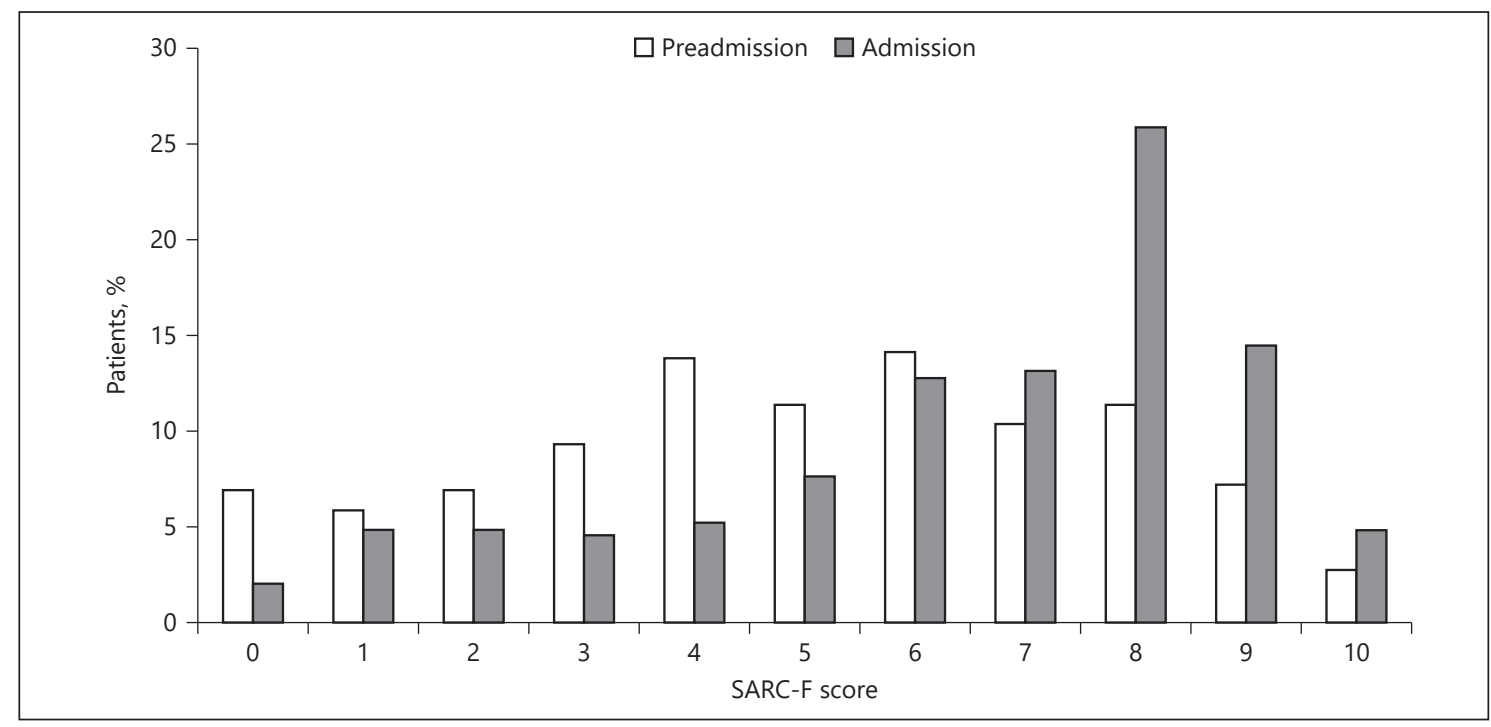

Fig. 2. Distribution of SARC-F scores at preadmission and admission in geriatric rehabilitation inpatients $(n=$ 290). SARC-F, Strength, Assistance in walking, Rise from a chair, Climb stairs, Falls history questionnaire.

Table 3. SARC-F (cut-off $\geq 4$ ) screening for preadmission and admission status and sarcopenia assessment at admission in geriatric rehabilitation inpatients

\begin{tabular}{|c|c|c|c|c|c|c|c|c|c|}
\hline \multirow[t]{2}{*}{ SARC-F } & \multicolumn{3}{|c|}{ EWGSOP, $n(\%)$} & \multicolumn{3}{|c|}{ EWGSOP2, $n(\%)$} & \multicolumn{3}{|c|}{ AWGS, $n(\%)$} \\
\hline & + & - & total & + & - & total & + & - & total \\
\hline \multicolumn{10}{|c|}{ Preadmission } \\
\hline$\geq 4$ & $66(27.2)$ & $99(40.7)$ & $165(67.9)$ & $46(18.9)$ & $115(47.1)$ & $161(66.0)$ & $64(27.0)$ & $96(40.5)$ & $160(67.5)$ \\
\hline$<4$ & $32(13.2)$ & $46(18.9)$ & $78(32.1)$ & $16(6.6)$ & $67(27.5)$ & $83(34.0)$ & $28(11.8)$ & $49(20.7)$ & 77 (32.5) \\
\hline Total & $98(40.3)$ & $145(59.7)$ & $243(100)$ & $62(25.4)$ & $182(74.6)$ & $244(100)$ & $92(38.8)$ & $145(61.2)$ & $237(100)$ \\
\hline \multicolumn{10}{|l|}{ Admission } \\
\hline$\geq 4$ & $74(33.2)$ & $109(48.9)$ & $183(82.1)$ & $46(20.8)$ & $133(60.2)$ & $179(81.0)$ & $68(31.3)$ & $109(50.2)$ & 177 (81.6) \\
\hline$<4$ & $19(8.5)$ & $21(9.4)$ & 40 (17.9) & $9(4.1)$ & $33(14.9)$ & $42(19.0)$ & $14(6.5)$ & $26(12.0)$ & $40(18.4)$ \\
\hline Total & $93(41.7)$ & $130(58.3)$ & $223(100)$ & $55(24.9)$ & $166(75.1)$ & $221(100)$ & $82(37.8)$ & $135(62.2)$ & $217(100)$ \\
\hline
\end{tabular}

SARC-F, Strength, Assistance in walking, Rise from a chair, Climb stairs, Falls history questionnaire. EWGSOP, European Working Group on Sarcopenia in Older People; AWGS, Asian Working Group for Sarcopenia.

and $56-80 \%$ with cognitive impairment) and poor specificity $(24-44 \%$ without and $17-39 \%$ with cognitive impairment) at both time points to identify inpatients at risk for sarcopenia compared to all 3 applied sarcopenia definitions. The survey completed without the patient resulted in fair to good sensitivity at preadmission (69-79\% without and $78-87 \%$ with cognitive impairment) and good sensitivity at admission $(92-100 \%$ without and $91-$ $100 \%$ with cognitive impairment). The specificity was poor (7-17\% without and $8-36 \%$ with cognitive impairment) at both time points. The AUC was poor to fair
(0.437-0.583 without and $0.378-0.600$ with cognitive impairment) at both time points, irrespective of who completed the SARC-F.

\section{Discussion}

In geriatric rehabilitation inpatients, the SARC-F showed poor diagnostic accuracy in identifying inpatients at risk for sarcopenia defined according to the EWGSOP, EWGSOP2, and AWGS definitions. 
Table 4. Diagnostic accuracy of SARC-F (cut-off $\geq 4$ ) at preadmission and admission for sarcopenia diagnosis at admission in geriatric rehabilitation inpatients

\begin{tabular}{|c|c|c|c|c|c|c|c|c|c|c|c|c|}
\hline \multirow[t]{2}{*}{ SARC-F } & \multicolumn{4}{|c|}{ EWGSOP } & \multicolumn{4}{|c|}{ EWGSOP2 } & \multicolumn{4}{|c|}{ AWGS } \\
\hline & $n$ & AUC (95\% CI) & Sens. & Spec. & $n$ & AUC (95\% CI) & Sens. & Spec. & $n$ & AUC (95\% CI) & Sens. & Spec. \\
\hline Preadmission & 243 & $0.495(0.421-0.570)$ & 0.673 & 0.317 & 244 & $0.555(0.474-0.636)$ & 0.742 & 0.368 & 237 & $0.517(0.411-0.592)$ & 0.696 & 0.338 \\
\hline Admission & 223 & $0.479(0.401-0.556)$ & 0.785 & 0.168 & 221 & $0.518(0.430-0.605)$ & 0.836 & 0.199 & 217 & $0.511(0.432-0.590)$ & 0.829 & 0.193 \\
\hline
\end{tabular}

SARC-F, Strength, Assistance in walking, Rise from a chair, Climb stairs, Falls history questionnaire. EWGSOP, European Working Group on Sarcopenia in Older People; AWGS, Asian Working Group for Sarcopenia; AUC, area under the curve; CI, confidence interval; Sens., sensitivity; Spec., specificity.

The fair to good sensitivity of the SARC-F in geriatric rehabilitation inpatients contrasts with the sensitivity reported in other settings. Poor (16-43\%) sensitivity was reported in geriatric hospitalized patients, geriatric outpatients $[15,17]$, and community-dwelling older adults (14-21\%) [34], although some studies in communitydwelling older adults also reported fair (78\%) sensitivity [13]. However, good sensitivity (95\%) was reported in older patients with hip fractures [16]. The poor specificity of the SARC-F in our population contrasts with the good specificity (up to 99\%) reported among community-dwelling [10,11,34], acute geriatric inpatients [15], geriatric outpatients [17], and nursing home populations $[18,19]$, and fair specificity in older patients with hip fractures [16]. The SARC-F had poor AUC values which is in contrast to the fair AUC for the acute geriatric ward (AUC 0.64 ) [15], older patients with hip fractures (AUC 0.5740.713 ) [16], and nursing homes (0.769-0.791) [19] whilst other studies reported poor [13], fair [12], and good [9, 14] diagnostic accuracy in community-dwelling older adults. These contrasting results may be influenced by different factors. First, although sensitivity and specificity are assumed to be intrinsically independent of the prevalence of the disease, it was suggested that the sensitivity and specificity might not be as unaffected by prevalence as thought [35]. It was previously found that the specificity decreases with higher disease prevalence [35], which is in accordance with the poor specificity and high prevalence we reported in geriatric rehabilitation inpatients. Second, different definitions of sarcopenia diagnosis were used, and moreover, within one definition, multiple measures can be used, for example, muscle strength can be measured by grip strength or chair stand test in EWGSOP2 [6]. Third, the phenotype of the assessed older adult highly depends on the population and setting, which implicates comparisons between populations. Diagnostic accuracy depends on patient characteristics and disease spectrum such as disease severity, stage, and co- morbidity [36]. For example, an acute event or accelerated chronic disease could lead to acute hospitalization of an older adult which may result in an acute decline in muscle mass and function and could result in sarcopenia, or at least a positive screening result on the SARC-F [37]. However, in the hospital setting, the presence of low muscle mass can be the cause or the consequence of the hospitalization. Since the diagnostic accuracy depends on multiple factors, such as prevalence, method of diagnosis, and patient characteristics, a large range of diagnostic accuracies can be found in the literature. The combination of fair to good sensitivity, poor specificity, and poor accuracy results in a negative advise for screening for sarcopenia by using the SARC-F in geriatric rehabilitation inpatients. Furthermore, the low correlation between SARC-F at the 2 time-points may be explained by the decrease in functional performance, leading up to the acute event that results in acute geriatric care.

Since sarcopenia is defined as low muscle mass combined with low muscle function $[6,20,22]$ and the SARC$\mathrm{F}$ is more sensitive to detect measures of muscle functioning compared to measures of muscle mass [10], it is not surprising that the SARC-F is not accurate in identifying inpatients at risk for sarcopenia. Also, the physical impairments as a result of the acute event leading to their geriatric rehabilitation admission may contribute to the positive SARC-F findings. As a result, alternative screening methods have been suggested in the literature. First, calf-circumference $[33,38,39]$, age, and being underweight [39] were added to the SARC-F, which could improve, in different studies, the sensitivity $[33,38,39]$. Second, optimizing the cut-off score to 3 or 6 points, respectively, improved diagnostic accuracy in acute geriatric wards, patients with hip fracture, and community-dwelling older people $[15,16,33]$. The optimal cut-off point of a tool depends on the best equilibrium between the sensitivity and specificity of the tool, which in its turn depends on the purpose of the screening tool. To prevent 
unneeded sarcopenia assessment (avoid false positives), a high specificity could be aimed for. In contrast, high sensitivity is the most important factor to initiate interventions for patients with sarcopenia and to avoid false negatives. Third, another screening tool, the Mini Sarcopenia Risk Assessment questionnaire, was developed. The Mini Sarcopenia Risk Assessment- 5 consists of 5 questions including weight loss and regularity of meals, resulting in higher sensitivity (80-90\%) compared to SARC-F while reducing the specificity $(60-80 \%)$ in community-dwelling older adults [40]. In conclusion, multiple ways of improving sarcopenia screening tools have been investigated, in order to improve diagnostic accuracy, as found in geriatric rehabilitation inpatients.

The SARC-F was originally designed to be completed by older adults themselves [5]. Research showed that, for example, when assessing the quality of life in older adults with dementia, the results depend on who completes the questionnaire, or the relationship between the completer and the older adult [41]. Overall, our results showed that there was no or only limited (higher sensitivity but lower specificity) impact on the diagnostic accuracy by who completed the survey, both in patients with or without cognitive impairment.

\section{Strengths and Limitations}

To the best of our knowledge, this is the first study investigating the diagnostic accuracy of the SARC-F to screen for sarcopenia in geriatric rehabilitation inpatients. Another strength of this study is that the SARC-F was validated against 3 different definitions of sarcopenia $[6,20,22]$ and reflected 2 time-points, preadmission and admission status. Furthermore, the likelihood of selection bias is low as the RESORT cohort is an inception cohort of all patients admitted to rehabilitation wards. One limitation is that the muscle mass for sarcopenia diagnosis was measured using BIA, which can be influenced by the hydration status. Also, the reliability to correctly complete a SARC-F questionnaire concerning a period 1 month earlier has not been studied yet. Last, an updated definition of the AWGS definition has been published after this study but was not included in this study [42].

\section{Conclusion}

The SARC-F has poor diagnostic accuracy and is therefore not valid for use in geriatric rehabilitation inpatients. As a result, the assessment of sarcopenia is recommended without screening.

Sarcopenia Screening Is Inaccurate in Geriatric Rehabilitation Inpatients

\section{Acknowledgements}

The authors thank the multidisciplinary team members of the Royal Melbourne Hospital, Royal Park Campus, involved in the RESORT cohort for their clinical work and the @AgeMelbourne team for their role in the data collection.

\section{Statement of Ethics}

The RESORT study was approved by the Melbourne Health Human Research Ethics Committee (HREC/17/MH/103) and performed in accordance to the Declaration of Helsinki. Written informed consent was obtained by the patient or a nominated proxy.

\section{Conflict of Interest Statement}

The authors have no conflict of interests to declare.

\section{Funding Sources}

This work was supported by the University of Melbourne (unrestricted grant received by Prof. Andrea B. Maier) and the Medical Research Future Fund (MRFF) provided by the Melbourne Academic Centre for Health (MACH). The funders had no role in the study design, data collection, and the preparation of this article.

\section{Author Contributions}

Lenore Dedeyne: conceptualization, methodology, formal analysis, and writing - original draft. Esmee M. Reijnierse: conceptualization, methodology, investigation, data curation, formal analysis, and writing - review and editing. Jacob Pacifico: investigation, data curation, and writing - review and editing. Jacqueline E. Kay: investigation, data curation, and writing - review and editing. Patricia Maggs: investigation, data curation, and writing - review and editing. Sabine Verschueren: supervision and writing review and editing. Jos Tournoy: supervision and writing - review and editing. Evelien Gielen: supervision and writing - review and editing. Wen Kwang Lim: investigation, data curation, and writing - review and editing. Andrea B. Maier: conceptualization, methodology, investigation, data curation, formal analysis, supervision, and writing - review and editing.

\section{References}

1 Sanchez-Rodriguez D, Marco E, Miralles R, Fayos M, Mojal S, Alvarado M, et al. Sarcopenia, physical rehabilitation and functional outcomes of patients in a subacute geriatric care unit. Arch Gerontol Geriatr. 2014 JulAug;59(1):39-43.

2 Zhang X, Wang C, Dou Q, Zhang W, Yang Y, Xie X. Sarcopenia as a predictor of all-cause mortality among older nursing home residents: a systematic review and meta-analysis. BMJ Open. 2018 Nov 12;8(11):e021252. 
3 Yeung SSY, Reijnierse EM, Pham VK, Trappenburg MC, Lim WK, Meskers CGM, et al. Sarcopenia and its association with falls and fractures in older adults: a systematic review and meta-analysis. J Cachexia Sarcopenia Muscle. 2019 Jun;10(3):485-500.

4 Wang DXM, Yao J, Zirek Y, Reijnierse EM, Maier AB. Muscle mass, strength, and physical performance predicting activities of daily living: a meta-analysis. J Cachexia Sarcopenia Muscle. 2020 Feb;11(1):3-25.

5 Malmstrom TK, Morley JE. SARC-F: a simple questionnaire to rapidly diagnose sarcopenia. J Am Med Dir Assoc. 2013 Aug;14(8):531-2.

6 Cruz-Jentoft AJ, Bahat G, Bauer J, Boirie Y, Bruyère $O$, Cederholm $T$, et al. Sarcopenia: revised European consensus on definition and diagnosis. Age Ageing. 2019 Jan 1;48(1):16-31.

7 Bauer J, Morley JE, Schols AMWJ, Ferrucci L, Cruz-Jentoft AJ, Dent E, et al. Sarcopenia: a time for action. An SCWD position paper. J Cachexia Sarcopenia Muscle. 2019 Oct;10(5):956-61.

8 Dent E, Morley JE, Cruz-Jentoft AJ, Arai H, Kritchevsky SB, Guralnik J, et al. International Clinical Practice Guidelines for Sarcopenia (ICFSR): screening, diagnosis and management. J Nutr Health Aging. 2018;22(10):1148-61.

9 Woo J, Leung J, Morley JE. Validating the SARC-F: a suitable community screening tool for sarcopenia? J Am Med Dir Assoc. 2014 Sep;15(9):630-4.

10 Bahat G, Yilmaz O, Kılıı̨ C, Oren MM, Karan MA. Performance of SARC-F in regard to sarcopenia definitions, muscle mass and functional measures. J Nutr Health Aging. 2018 2018/10/01;22(8):898-903.

11 Kera T, Kawai H, Hirano H, Kojima M, Watanabe $\mathrm{Y}$, Motokawa $\mathrm{K}$, et al. SARC-F: a validation study with community-dwelling older Japanese adults. Geriatr Gerontol Int. 2019 Nov;19(11):1172-8.

12 Hajaoui M, Locquet M, Beaudart C, Reginster JY, Petermans J, Bruyère O. Sarcopenia: performance of the SARC-F questionnaire according to the European consensus criteria, EWGSOP1 and EWGSOP2. J Am Med Dir Assoc. 2019 Sep;20(9):1182-3.

13 Sanchez-Rodriguez D, Marco E, DavalosYerovi V, Lopez-Escobar J, Messaggi-Sartor $\mathrm{M}$, Barrera C, et al. Translation and validation of the Spanish version of the SARC-F questionnaire to assess sarcopenia in older people. J Nutr Health Aging. 2019;23(6):518-24.

14 Yang M, Hu X, Xie L, Zhang L, Zhou J, Lin J, et al. Comparing mini sarcopenia risk assessment with SARC-F for screening sarcopenia in community-dwelling older adults. J Am Med Dir Assoc. 2019 Jan;20(1):53-7.

15 Li M, Kong Y, Chen H, Chu A, Song G, Cui Y. Accuracy and prognostic ability of the SARCF questionnaire and Ishii's score in the screening of sarcopenia in geriatric inpatients. Braz J Med Biol Res. 2019;52(9):e8204.

16 Ha YC, Won C, Kim M, Chun KJ, Yoo JI. SARC-F as a useful tool for screening sarcopenia in elderly patients with hip fractures. J Nutr Health Aging. 2020;24(1):78-82.
17 Mienche M, Setiati S, Setyohadi B, Kurniawan J, Laksmi PW, Ariane A, et al. Diagnostic performance of calf circumference, thigh circumference, and SARC-F questionnaire to identify sarcopenia in elderly compared to Asian working group for sarcopenia's diagnostic standard. Acta Med Indones. 2019 Apr; 51(2):117-27.

18 Kotlarczyk MP, Perera S, Nace DA, Resnick NM, Greenspan SL. Identifying sarcopenia in female long-term care residents: a comparison of current guidelines. J Am Geriatr Soc. 2018 Feb;66(2):316-20.

19 Yang M, Lu J, Jiang J, Zeng Y, Tang H. Comparison of four sarcopenia screening tools in nursing home residents. Aging Clin Exp Res. 2019 Oct;31(10):1481-9.

20 Cruz-Jentoft AJ, Baeyens JP, Bauer JM, Boirie Y, Cederholm T, Landi F, et al. Sarcopenia: European consensus on definition and diagnosis: report of the European Working Group on Sarcopenia in Older People. Age Ageing. 2010 Jul;39(4):412-23.

21 Zanker J, Scott D, Reijnierse EM, BrennanOlsen SL, Daly RM, Girgis CM, et al. Establishing an operational definition of sarcopenia in Australia and New Zealand: delphi method based consensus statement. J Nutr Health Aging. 2019;23(1):105-10.

22 Chen LK, Liu LK, Woo J, Assantachai P, Auyeung TW, Bahyah KS, et al. Sarcopenia in Asia: consensus report of the Asian Working Group for Sarcopenia. J Am Med Dir Assoc. 2014 Feb;15(2):95-101.

23 Chumlea WC, Roche AF, Steinbaugh ML. Estimating stature from knee height for persons 60 to 90 years of age. J Am Geriatr Soc. 1985 Feb;33(2):116-20.

24 Rockwood K, Song X, MacKnight C, Bergman H, Hogan DB, McDowell I, et al. A global clinical measure of fitness and frailty in elderly people. CMAJ. 2005 Aug 30;173(5): 489-95.

25 Charlson ME, Pompei P, Ales KL, MacKenzie CR. A new method of classifying prognostic comorbidity in longitudinal studies: development and validation. J Chronic Dis. 1987; 40(5):373-83

26 Ferguson M, Capra S, Bauer J, Banks M. Development of a valid and reliable malnutrition screening tool for adult acute hospital patients. Nutrition. 1999 Jun;15(6):45864.

27 Katz S, Ford AB, Moskowitz RW, Jackson BA, Jaffe MW. Studies of illness in the aged. The index of Adl: a standardized measure of biological and psychosocial function. JAMA. 1963 Sep 21;185:914-9.

28 Lawton MP, Brody EM. Assessment of older people: self-maintaining and instrumental activities of daily living. Gerontologist. 1969 Autumn;9(3):179-86.

29 Ling $\mathrm{CH}$, de Craen AJ, Slagboom PE, Gunn DA, Stokkel MP, Westendorp RG, et al. Accuracy of direct segmental multi-frequency bioimpedance analysis in the assessment of total body and segmental body composition in middle-aged adult population. Clin Nutr. 2011 Oct;30(5):610-5.

30 Janssen I, Baumgartner RN, Ross R, Rosenberg IH, Roubenoff R. Skeletal muscle cutpoints associated with elevated physical disability risk in older men and women. Am J Epidemiol. 2004 Feb 15;159(4):413-21.

31 Reijnierse EM, de Jong N, Trappenburg MC, Blauw GJ, Butler-Browne G, Gapeyeva H, et al. Assessment of maximal handgrip strength: how many attempts are needed? J Cachexia Sarcopenia Muscle. 2017 Jun;8(3):466-74.

32 Guralnik JM, Simonsick EM, Ferrucci L, Glynn RJ, Berkman LF, Blazer DG, et al. A short physical performance battery assessing lower extremity function: association with self-reported disability and prediction of mortality and nursing home admission. J Gerontol. 1994 Mar;49(2):M85-94.

33 Barbosa-Silva TG, Menezes AM, Bielemann RM, Malmstrom TK, Gonzalez MC, Grupo de Estudos em Composicao Corporal e $\mathrm{Nu}$ trição. Enhancing SARC-F: improving sarcopenia screening in the clinical practice. J Am Med Dir Assoc. 2016 Dec 1;17(12):1136-41.

34 Ida S, Kaneko R, Murata K. SARC-F for screening of sarcopenia among older adults: a meta-analysis of screening test accuracy. J Am Med Dir Assoc. 2018 Aug; 19(8):685-9.

35 Leeflang MM, Rutjes AW, Reitsma JB, Hooft L, Bossuyt PM. Variation of a test's sensitivity and specificity with disease prevalence. CMAJ. 2013 Aug 6;185(11):E537-44.

36 Simundic AM. Measures of diagnostic accuracy: basic definitions. EJIFCC. 2009 Jan; 19(4):203-11.

37 Welch C, K. Hassan-Smith Z, A. Greig C, M. Lord J, T. Jackson T. Acute sarcopenia secondary to hospitalisation: an emerging condition affecting older adults. Aging Dis. 2018 Feb;9(1):151-64.

38 Yang M, Hu X, Xie L, Zhang L, Zhou J, Lin J, et al. Screening sarcopenia in communitydwelling older adults: SARC-F vs SARC-F combined with calf circumference (SARCCalF). J Am Med Dir Assoc. 2018 Mar;19(3): e1-277.

39 Kurita N, Wakita T, Kamitani T, Wada O, Mizuno K. SARC-F validation and SARC$\mathrm{F}+\mathrm{EBM}$ derivation in musculoskeletal disease: the SPSS-OK study. J Nutr Health Aging. 2019;23(8):732-8.

40 Yang M, Hu X, Xie L, Zhang L, Zhou J, Lin J, et al. Validation of the Chinese version of the mini sarcopenia risk assessment questionnaire in community-dwelling older adults. Medicine. 2018 Sep;97(37):e12426.

41 Griffiths AW, Smith SJ, Martin A, Meads D, Kelley R, Surr CA. Exploring self-report and proxy-report quality-of-life measures for people living with dementia in care homes. Qual Life Res. 2020 Feb;29(2):463-72.

42 Chen LK, Woo J, Assantachai P, Auyeung TW, Chou MY, Iijima K, et al. Asian Working Group for Sarcopenia: 2019 consensus update on sarcopenia diagnosis and treatment. J Am Med Dir Assoc. 2020;21(3):300-e2. 